\title{
Attitudes et croyances des parents au sujet du port obligatoire du casque de vélo : comparaison entre provinces avec et sans législation
}

\author{
P. C. Parkin, M.D. (1, 2, 3); J. DeGroot, M. Sc. (1, 2); A. Macpherson, Ph. D. (4); P. Fuselli, M. Sc. (5); C. Macarthur, \\ $\operatorname{MBChB}(1,2,3)$
}

Cet article a fait l'objet d'une évaluation par les pairs.

\section{Résumé}

Introduction : L’objectif de l'étude était d'effectuer un sondage auprès de parents canadiens sur leurs attitudes et leurs croyances au sujet de la législation du port du casque chez les cyclistes et de comparer les réponses des parents vivant dans une province ayant légiféré dans ce domaine et celles de ceux vivant dans une province n’ayant pas légiféré.

Méthodologie : Un sondage national a été réalisé auprès de 1002 parents d'enfants âgés de moins de 18 ans. Des tests du chi-carré ont été utilisés pour comparer les réponses des parents dans les différentes provinces.

Résultats : Les réponses des parents vivant dans une province ayant légiféré $(\mathrm{n}=640)$ ou ne l'ayant pas fait $(n=362)$ s'établissent respectivement comme suit : sont préoccupés par les accidents de vélo (63\% et $68 \%$, non significatif [NS]); croient que le port du casque de vélo est une mesure efficace (98\% et $98 \%$, NS); l'enfant porte toujours un casque de vélo (74 \% et $69 \%$, NS); sont favorables à une loi visant les enfants $(95 \%$ et $83 \%, p<0,001)$; sont favorables à une loi visant les cyclistes de tous âges $(85 \%$ et $75 \%, p<0,001)$; sont favorables à l'application de la loi par les services de police ( $83 \%$ et $76 \%, p=0,003$ ); croient que la loi réduit la durée d'utilisation de la bicyclette par leur enfant (5\% et $8 \%$, NS).

Conclusion : Les parents sont largement favorables à la législation du port du casque chez les cyclistes au Canada. Ils croient que le casque de vélo est une mesure efficace et que la loi n’a pas pour effet de réduire la durée d'utilisation de la bicyclette par leur enfant. Ils sont également largement favorables à une loi visant les cyclistes de tous âges et à l'application de la loi par les services de police.

Mots-clés : casque, législation, sondages, enfant, attitude, santé publique, dispositifs de protection de la tête, vélo

\section{Introduction}

Des études systématiques révèlent que le port du casque par les cyclistes réduit le risque de lésions crâniennes, cérébrales et faciales et que la législation du port favorise l'usage de la bicyclette et réduit les taux de blessures à la tête ${ }^{1-3}$. Plusieurs provinces canadiennes (6 sur 10) ont adopté une loi sur le port du casque, et certaines municipalités ont mis en place des textes législatifs plus stricts et ayant une portée plus universelle ${ }^{4}$. Malgré des données probantes favorables, le débat sur les avantages du port du casque et sa législation se poursuit ${ }^{5,6}$. Ce débat, toutefois, n’a pas encore été mené dans une perspective sociétale.

Notre étude visait à réaliser un sondage auprès de parents canadiens sur leurs attitudes et leurs croyances au sujet de la législation du port du casque de vélo et à effectuer des comparaisons entre ceux qui habitent dans une province ayant légiféré dans ce domaine et ceux qui habitent dans une province ne l'ayant pas fait.

\section{Méthodologie}

Nous avons conçu notre sondage afin de pouvoir traiter de plusieurs enjeux du point de vue des parents canadiens. Nous avons posé des questions sur la perception des parents à l'égard de l'efficacité du casque de vélo, sur leur soutien à une législation rendant obligatoire le port du casque par les cyclistes et sur leurs perceptions concernant l'influence de la loi sur l'usage de la bicyclette. Nous avons également recueilli des données sur les répondants, notamment l'âge et le sexe de leurs enfants, l'âge et la scolarité du parent répondant, le revenu du ménage et la province de résidence de la famille. Le sondage a été réalisé entre le $1^{\text {er }}$ et le 5 février 2010. Le cadre d'échantillonnage

Rattachement des auteurs :

1. Division of Paediatric Medicine and the Paediatric Outcomes Research Team (PORT), Hospital for Sick Children, Toronto (Ontario), Canada

2. Child Health Evaluative Sciences, Hospital for Sick Children, Toronto (Ontario), Canada

3. Department of Pediatrics and Institute of Health Policy, Management and Evaluation, University of Toronto Faculty of Medicine, Toronto (Ontario), Canada

4. School of Kinesiology and Health Science, York University, Toronto (Ontario), Canada

5. SécuriJeunes Canada, Toronto (Ontario), Canada

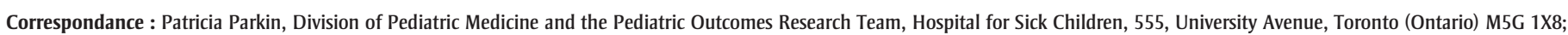
tél. : 416-813-6933; téléc. : 416-813-5663; courriel : patricia.parkin@sickkids.ca 
était constitué d'adultes canadiens âgés de 18 ans et plus membres du panel LegerWeb*. Ce panel national, qui est utilisé pour mener environ 1000 sondages par an au Canada, se compose de 345000 membres; de 10000 à 20000 nouveaux membres s'y ajoutent chaque mois et le taux de rétention des membres est de $90 \%$. Le recrutement des nouveaux panélistes se fait par invitation, afin de garantir une bonne représentativité de la population adulte du Canada selon le sexe, l'âge, le revenu et la région. Pour encourager la participation, les répondants sont inscrits à des tirages au sort mensuels qui offrent des prix. Pour l'étude présentée ici, des panélistes ayant des enfants âgés de moins de 18 ans ont été choisis au hasard et ont reçu un courriel les invitant à participer au sondage.

Un échantillon de 1000 répondants était suffisant pour déterminer la proportion de répondants favorables à l'adoption d'une loi, avec une marge d'erreur de plus ou moins $3 \%$ avec un intervalle de confiance (IC) à $95 \%$, afin d'obtenir une puissance de détection de $90 \%$ pour un écart de $10 \%$ entre les répondants vivant dans une province ayant légiféré et ceux vivant dans une province ne l'ayant pas fait. Nous avons utilisé des statistiques descriptives pour présenter les réponses de l'ensemble de la population visée par le sondage et des tests du chi carré pour comparer les répondants vivant dans une province ayant légiféré dans le domaine à ceux vivant dans une province ne l'ayant pas fait. Nous avons appliqué la correction de Bonferroni pour tenir compte des comparaisons multiples (une valeur $p$ corrigée inférieure à 0,004 est considérée comme significative). Nous avons également effectué une analyse exploratoire des réponses de ceux vivant dans une province imposant le port du casque aux cyclistes de tous âges par rapport à ceux vivant dans une province où cette obligation ne touche que les enfants.

Le comité d'éthique de la recherche du Hospital for Sick Children a approuvé l'étude.

\section{Résultats}

Sur les 1128 parents invités à participer au sondage, 1002 ont répondu (pour un taux de réponse de $89 \%$ ) : 640 provenaient de provinces ayant légiféré (155 avec loi visant tous les cyclistes et 485 avec loi ne visant que les enfants) et 362 de provinces n'ayant pas légiféré dans ce domaine. Seulement 3,6 \% des répondants ont déclaré que leur(s) enfant(s) avai(en)t souffert d'une blessure à bicyclette exigeant des soins médicaux. Les caractéristiques des parents répondants et de leurs enfants sont présentées dans le tableau 1. La proportion de répondants dont le revenu du ménage se situait entre 50000 \$ et $125000 \$(53 \%$, IC à $95 \%$ : $50 \%$ à $56 \%$ ) est analogue à celle du recensement national pour le revenu familial $(51 \%)^{7}$. La proportion de répondants ayant poursuivi des études universitaires (50\%, IC à $95 \%$ : $47 \%$ à $53 \%$ ) est supérieure à celle $\mathrm{du}$ recensement national pour les adultes de 25 à 64 ans $(23 \%)^{8}$. La proportion de répondants par province est analogue à la densité de la population par province fournie par les données du recensement national ${ }^{9}$.

Les parents vivant dans une province ayant légiféré ont répondu aux diverses questions posées comme suit : sont préoccupés par les accidents de vélo, $63 \%$ (contre $68 \%$ pour les parents vivant dans une province n'ayant pas légiféré, non significatif [NS]); croient que le port du casque de vélo est une mesure efficace, $98 \%$ (contre $98 \%$, NS); l'enfant porte toujours un casque lorsqu'il fait du vélo, $74 \%$ (contre $69 \%$, NS); sont favorables à une loi imposant le port du casque à tous les enfants, $95 \%$ (contre $83 \%$, $p<0,001$ ); sont favorables à une loi imposant le port du casque aux cyclistes de tous âges, $85 \%$ (contre $75 \%, p<0,001$ ); sont favorables à l'application de la loi par les services de police, $83 \%$ (contre $76 \%$, $p=0,003$ ); croient que la loi réduit la durée d'utilisation de la bicyclette par leur enfant, $5 \%$ (contre $8 \%$, NS).

Les parents vivant dans une province imposant le port du casque à tous les cyclistes ont répondu comme suit par rapport à ceux vivant dans une province où seuls les enfants doivent porter le casque : sont préoccupés par les accidents de vélo, $68 \%$ contre $61 \%$; croient que le port du casque est une mesure efficace, $96 \%$ contre $99 \%$; l'enfant porte toujours un casque lorsqu'il fait du vélo, $77 \%$ contre $73 \%$; sont favorables à une loi visant uniquement les enfants, $97 \%$ contre $94 \%$; sont favorables à une loi visant les cyclistes de tous âges, $91 \%$ contre $84 \%$; sont favorables à l'application de la loi par les services de police, $89 \%$ contre $82 \%$; croient que la loi réduit la durée d'utilisation de la bicyclette par leur enfant $6 \%$ contre $5 \%$. Aucune de ces comparaisons n'est statistiquement significative (soit $p<0$,004).

\section{Analyse}

Cet échantillon national révèle que de nombreux parents croient que le port du casque est une mesure efficace et que l'adoption d'une loi en la matière ne réduit pas la durée d'utilisation de la bicyclette par leur enfant. De nombreux parents favorisent également l'adoption d'une loi touchant tous les cyclistes et l'application de cette loi par les services de police.

Un sondage antérieur réalisé en 1991 dans une ville canadienne avant l'adoption d'une loi dans ce domaine révélait un taux d'appui de $80 \%$ en faveur de l'adoption de cette $\operatorname{loi}^{10}$. Ce chiffre est comparable au taux d'appui que nous avons observé chez les parents vivant dans une province n'ayant pas de loi dans le domaine. Le taux d'appui actuel de $93 \%$ observé chez les parents vivant dans une province ayant une loi dans le domaine met en évidence une augmentation importante du taux d'appui au cours des deux dernières décennies.

Quatre provinces canadiennes sur dix (Colombie-Britannique, NouveauBrunswick, île-du-Prince-Édouard et NouvelleÉcosse) ont une législation imposant le port du casque à l'ensemble des cyclistes; la législation en Alberta et en Ontario vise les cyclistes âgés de moins de 18 ans; les autres

\footnotetext{
${ }^{*}$ http://www.legermarketing.com
} 
TABLEAU 1

Caractéristiques des participants au sondage $(\mathrm{N}=1002)$

\begin{tabular}{|c|c|c|}
\hline Variables & n & $(\%)$ \\
\hline \multicolumn{3}{|l|}{ Âge des parents (ans) } \\
\hline moins de 35 & 375 & $(37,4)$ \\
\hline 35 à 44 & 467 & $(46,6)$ \\
\hline 45 et plus & 160 & $(16,0)$ \\
\hline \multicolumn{3}{|l|}{ Sexe des parents sondés } \\
\hline hommes & 465 & $(46,4)$ \\
\hline \multicolumn{3}{|l|}{ Scolarité des parents } \\
\hline études secondaires / collégiales & 492 & $(49,1)$ \\
\hline université & 500 & $(49,9)$ \\
\hline préfère ne pas répondre & 10 & $(1,0)$ \\
\hline \multicolumn{3}{|l|}{ Revenu du ménage (dollars) } \\
\hline moins de 50000 & 178 & $(17,8)$ \\
\hline 50000 à 124999 & 528 & $(52,7)$ \\
\hline 125000 et plus & 160 & $(16,0)$ \\
\hline ne sait pas / préfère ne pas répondre & 136 & $(13,6)$ \\
\hline \multicolumn{3}{|l|}{ Âge des enfants (ans) ${ }^{a}$} \\
\hline moins de 1 & 138 & $(6,8)$ \\
\hline 1 à 4 & 829 & $(40,8)$ \\
\hline 5 à 9 & 777 & $(38,2)$ \\
\hline 10 à 14 & 217 & $(10,7)$ \\
\hline 15 à 17 & 72 & $(3,5)$ \\
\hline \multicolumn{3}{|l|}{ Sexe des enfants } \\
\hline masculin seulement & 292 & $(29,1)$ \\
\hline féminin seulement & 286 & $(28,5)$ \\
\hline masculin et féminin & 414 & $(41,3)$ \\
\hline préfère ne pas répondre & 10 & $(1,0)$ \\
\hline \multicolumn{3}{|l|}{ Province } \\
\hline Colombie-Britannique & 100 & $(10,0)$ \\
\hline Alberta & 85 & $(8,5)$ \\
\hline Saskatchewan & 28 & $(2,8)$ \\
\hline Manitoba & 57 & $(5,7)$ \\
\hline Ontario & 400 & $(39,9)$ \\
\hline Québec & 267 & $(26,7)$ \\
\hline Nouveau-Brunswick & 31 & $(3,1)$ \\
\hline Île-du-Prince-Édouard & 1 & $(0,1)$ \\
\hline Nouvelle-Écosse & 23 & $(2,3)$ \\
\hline Terre-Neuve-et-Labrador & 10 & $(1,0)$ \\
\hline \multicolumn{3}{|c|}{ L'enfant a eu un accident de vélo exigeant des soins médicaux } \\
\hline oui & 36 & $(3,6)$ \\
\hline non & 957 & $(95,5)$ \\
\hline ne sait pas / préfère ne pas répondre & 9 & $(0,9)$ \\
\hline
\end{tabular}

a Si le nombre total d'enfants est supérieur au nombre de participants, c'est qu'une même famille peut compter plusieurs enfants $(n=2033)$.

provinces (Saskatchewan, Manitoba, Québec, Terre-Neuve-et-Labrador) ainsi que les territoires (Yukon, Territoires du Nord-Ouest et Nunavut) n'ont aucune législation dans ce domaine. Cette diversité offre de la matière à une expérience naturelle examinant l'usage du casque et les croyances qui s'y rattachent. Une analyse récente de données provenant de l'Enquête sur la santé dans les collectivités canadiennes révèle que l'usage autodéclaré du casque chez les jeunes (12 à 18 ans) augmente dans la mesure où la loi a une portée plus universelle : $33 \%$ dans les provinces n'ayant pas de législation, $47 \%$ dans les provinces où la législation ne vise que les enfants et $78 \%$ dans les provinces où les cyclistes de tous âges sont visés ${ }^{11}$. Dans notre groupe d'âge, composé principalement de préadolescents (86 \% avaient moins de 10 ans), l'universalité de la loi est associée à l'appui déclaré par les parents en faveur d'une loi (enfants uniquement et tous les cyclistes) et de son application par les services de police, mais non aux taux déclarés par les parents d'utilisation du casque par l'enfant.

En Ontario, l'une des deux provinces où la loi ne touche que les enfants, les autorités se sont demandé si la législation ne devrait pas s'appliquer à tous les cyclistes. En juin 2012, le Bureau du coroner en chef $^{12}$ a rendu compte de l'examen de 129 décès accidentels de cyclistes survenus en Ontario entre 2006 et 2010. De ce nombre, $15 \%$ avaient 19 ans ou moins et seulement $27 \%$ portaient un casque protecteur. Le rapport du coroner recommandait de modifier le code de la route afin de rendre obligatoire le port du casque chez les cyclistes de tous âges ${ }^{12}$. Les résultats de notre sondage donnent à penser que les parents accorderaient volontiers leur appui à une telle recommandation.

L'un des éléments du débat qui a cours actuellement sur les avantages et les inconvénients liés à l'adoption de textes législatifs rendant obligatoire le port du casque de vélo est que " la mise à exécution des lois décourage l'usage de la bicyclette et, partant, fait augmenter les coûts liés à l'obésité et au manque d'exercice pour la société et réduit la sécurité globale de l'usage de la bicyclette » [traduction] $^{13, p .86}$. Toutefois, l'observation directe d'enfants faisant de la bicyclette dans une ville canadienne chaque année entre 1993 et 1999 a révélé que l'adoption d'une loi sur le port du casque n'a pas eu d'effet notable sur la durée d'utilisation de la bicyclette par les enfants ${ }^{14}$. En outre, notre sondage révèle 
que $5 \%$ seulement des parents qui vivent dans une province où il $\mathrm{y}$ a une législation sur le port du casque ont déclaré que cette loi avait réduit le temps que leur enfant consacrait à faire de la bicyclette. Prises ensemble, toutes ces études sur les comportements d'enfants directement observés ou déclarés par les parents donnent à penser que la mise en place d'une loi a favorisé la sécurité sans pour autant réduire l'activité physique.

\section{Limites}

Le présent sondage comporte des limites liées à la scolarité plus élevée des parents répondants par rapport aux données du recensement national. Néanmoins, le fait que $70 \%$ des parents sondés aient indiqué que leur(s) enfant(s) porte(nt) toujours un casque correspond à ce qui a été directement observé dans les études sur des cyclistes réalisées dans les provinces canadiennes avant et après l'adoption d'une $1 i^{3,15,16}$. Par exemple, plusieurs années après l'adoption d'une loi en Alberta et en Nouvelle-Écosse, entre 63 et $90 \%$ des enfants et des adolescents roulant à vélo portaient un casque. Même si ces études, qui sont fondées sur l'observation directe, ne permettent pas d'évaluer la scolarité des parents, les sites d'observation ont été choisis au hasard et l'analyse a fait l'objet de contrôles du point de vue du quintile de revenu du voisinage. Par contraste, des observations directes sur le port du casque chez des enfants, six ans après l'adoption de la loi en Ontario, révèlent des variations en fonction du niveau de revenu du voisinage $^{17}$. Il est donc possible que les attitudes et les croyances des parents au sujet de la réglementation par la loi du port du casque soient influencées par leur scolarité et leur revenu.

Notre étude comporte également plusieurs autres limites potentielles. Par exemple, les données ont été recueillies au mois de février, une période peu propice à l'usage de la bicyclette chez les enfants. Il se pourrait que la sensibilité des parents au port du casque chez les enfants, que leur préoccupation à l'égard des accidents de vélo et que leur appui en faveur d'une loi soient plus élevés pendant les saisons au cours desquelles les enfants font habituel- lement de la bicyclette. Dans l'affirmative, les estimations contenues dans notre étude seraient dites prudentes. En outre, même si nous avons obtenu un taux de réponse élevé, nous ne disposons pas de données sur les non-répondants. Enfin, nous sommes conscients que seuls des parents ont répondu au sondage, et que d'autres membres de la société devraient pouvoir participer à ce débat, en particulier lorsqu'il s'agit de déterminer si la législation devrait être restreinte aux enfants ou s'appliquer à l'ensemble des cyclistes.

\section{Conclusion}

Au Canada, les parents sont largement favorables à la législation du port du casque de vélo. Notre étude offre une perspective sociétale susceptible d'éclairer le débat actuel et de jouer un rôle utile auprès des professionnels de la santé publique, des spécialistes du transfert des connaissances ainsi que des décideurs, que ce soit au Canada ou dans d'autres pays.

\section{Références}

1. Ivers R. Systematic reviews of bicycle helmet research. Inj Prev. 2007;13:190.

2. Thompson DC, Rivara FP, Thompson R. Helmets for preventing head and facial injuries in bicyclists. Cochrane Database Syst Rev. 1999;4:CD001855.

3. Macpherson A, Spinks A. Bicycle helmet legislation for the uptake of helmet use and prevention of head injuries. Cochrane Database Syst Rev. 2007;2:CD005401.

4. Karkhaneh M, Rowe BH, Saunders LD, Voaklander DC, Hagel BE. Bicycle helmet use after the introduction of all ages helmet legislation in an urban community in Alberta, Canada. Can J Public Health. 2011;102:134-8.

5. Robinson DL. No clear evidence from countries that have enforced the wearing of helmets. BMJ. 2006;332:722-5.

6. Hagel B, Macpherson A, Rivara FP, Pless B. Arguments against helmet legislation are flawed. BMJ. 2006;332:725-6.
7. Statistique Canada. Tableau 2 : Nombre et proportion des personnes de 25 à 64 ans selon le niveau de scolarité atteint et les groupes d'âge, Canada, 2006 [Internet]. Ottawa (Ont.) : Statistique Canada; 2006. Consultable à la page : http://www12.statcan .gc.ca/census-recensement/2006/as-sa/97 $-560 /$ table/t2-fra.cfm

8. Statistique Canada. Revenu familial, selon le type de famille (familles comptant un couple), 2009 [Internet]. Ottawa (Ont.) : Statistique Canada; 2009. Consultable à la page : http://www.statcan.gc.ca/tables -tableaux/sum-som/102/cst01/famil106a -fra.htm

9. Statistique Canada. Chiffres de population et des logements, Canada, provinces et territoires, recensements de 2011 et 2006 [Internet]. Ottawa (Ont.) : Statistique Canada; [modifié le 30 janvier 2013; consulté le 7 février 2013]. Consultable à la page : http://www12.statcan.gc.ca/census -recensement/2011/dp-pd/hlt-fst/pd-pl/Table -Tableau.cfm?Lang $=$ fra $\& T=101 \& S=50 \& O=A$

10. Hu X, Wesson DE, Parkin PC, Chipman ML, Spence LJ. Parental attitudes toward legislation for helmet use by child cyclists. Can J Public Health. 1993;84:163-5.

11. Dennis J, Potter B, Ramsay T, Zarychanski R. The effects of provincial bicycle helmet legislation on helmet use and bicycle ridership in Canada. Inj Prev. 2010;16:219-24.

12. Ministère de la sécurité communautaire et des services correctionnels de l'Ontario. Examen de tous les décès dus à un accident de vélo survenus en Ontario entre le $1^{\mathrm{er}}$ janvier 2006 et le 31 décembre 2010. Toronto (Ont.) : Bureau du coroner en chef de l'Ontario; juin 2012. Consultable à la page : http://www.mcscs.jus.gov.on.ca/ french/DeathInvestigations/office_coroner/ PublicationsandReports/accidentdevelo/DI_ Cycling_Death_Review_fr.html

13. Robinson DL. Bicycle helmet legislation: can we reach a consensus? Accid Anal Prev. 2007;9:86-93.

14. Macpherson AK, Parkin PC, To TM. Mandatory helmet legislation and children's exposure to cycling. Inj Prev. 2001;7:228-30. 
15. Karkhaneh M, Rowe BH, Saunders LD, Voaklander DC, Hagel BE. Bicycle helmet use four years after the introduction of helmet legislation in Alberta, Canada. Accid Anal Prev. 2011;43:788-96.

16. LeBlanc JC, Beattie TL, Culligan C. Effect of legislation on the use of bicycle helmets. CMAJ. 2002;166:592-5.

17. Macpherson AK, Macarthur C, To TM, Chipman ML, Wright JG, Parkin PC. Economic disparity in bicycle helmet use by children six years after the introduction of legislation. Inj Prev. 2006;12:231-5. 